\title{
Effects of Polysaccharide from Hericium Erinaceus on Diarrhea Rats Induced by Lincomycin Hydrochloride: A Potential Treatment for Gut Microbiota Dysbiosis
}

\section{Shanshan Li}

Jilin Agricultural Science and Technology University

Duoduo Ren

Chinese Academy of Agricultural Sciences

Shaochao Tan

Yantai Penglai Traditional Chinese Medicine Hospital

\section{Yuli Qi}

Chinese Academy of Agricultural Sciences

Yinshi Sun ( $\nabla$ sunyinshi2015@163.com )

Chinese Academy of Agricultural Sciences

\section{Research Article}

Keywords: Hericium erinaceus, polysaccharide, antibiotic-associated diarrhea, gut microbiota, butyrate

Posted Date: March 1st, 2022

DOI: https://doi.org/10.21203/rs.3.rs-1350856/v1

License: (c) (i) This work is licensed under a Creative Commons Attribution 4.0 International License.

Read Full License 


\section{Abstract}

In China, the fungus Hericium erinaceus is known as a healthy food crop which possesses digestive tract protection functionality. This research was focus on the effects of polysaccharides extracted from Hericium erinaceus (WHP) on rats with diarrhea induced by antibiotic, specifically on the shifts of gut microbiota. WHP was mainly composed of glucose (88.6\%), galactose $(9.1 \%)$, and fructose $(2.3 \%)$. It decreased the disease activity index, structure damage of the colon in rats with AAD; increased butyrate production, and affected the gut microbiota through increase the relative abundance of Lactobacillus and Butyricicoccus, while decreased that of Allobaculum and Enterococcus, which closely related to the gut microbiota dysbiosis. It could concluded from this study that WHP can ameliorate antibiotic induced diarrhea by causing the recovery of the colon damage, improving the gut microbiota dysbiosis, and promoting the butyrate production. The results of this study can provide valuable data on how Hericium erinaceus polysaccharide alleviate the damage associated to the intake of antibiotics.

\section{Introduction}

In China, the edible fungus Hericium erinaceus which serve as food is popular for its medicinal potency. The fruiting bodies have attracted a lot of attention in the medical, biology and food sciences field because of its multiple activities. It often used as home remedy, especially in soup, to ameliorate the chronic atrophic gastritis or other disease associated with the digestive tract. The polysaccharide is one of the major components in Hericium erinaceus, and series of reports have been published about the purification, fine structural analysis and pharmacological activities of this polysaccharide present in this fungus ${ }^{1,2}$. It has been reported that the method of extraction of this polysaccharide to affect its potency. Polysaccharide from the Hericium erinaceus extracted with citric acid had more obvious antioxidant capacities than when water and $0.9 \%$ sodium chloride was used as an extraction solvent in vitro ${ }^{3}$. The polysaccharide from fungal mycelium of Hericium erinaceus was investigated to have anti-gastritis, antiulcerative colitis, hepato-protective, antioxidant, and anti-gastric ulcer activities ${ }^{4}$, it also could prevent the apoptosis of gastric mucosal epithelial cell through apoptotic cellular signals induced by hydrogen peroxide ${ }^{5}$. Polysaccharides from the Hericium erinaceus fruiting body possess gastro-protective activity, which were related to the its ability of regulate the gastric secretions, improve the antioxidant status, and increase the release of defensive factors ${ }^{6}$, such as the $\beta$-glucan from the fruiting body which could modulate the digestibility of wheat-starch in vitro $^{7}$; the polysaccharide HP-ES could induce the dendritic cells activation and modulate the $T_{H} 1$ immune response to play an immuno-modulating function ${ }^{8}$; the polysaccharide HECP and HCRP could promote the colon health through improving the production of short chain fatty acids (SCFAs), adjusting the $\mathrm{pH}$ values and moisture levels of the colon contents, cecum contents and feces ${ }^{9}$; the polysaccharide HECP could ameliorate the mucosal barrier oxidative damage, improve the distribution of the intestinal flora in mice with colitis ${ }^{10}$; the polysaccharide $\mathrm{HEP}_{\mathrm{W} 1}$ showed positive effects on hydrogen peroxide-induced damage in the epithelial cells of the human gastric 5 . 
Antibiotics have been widely used as an important means of fighting against infectious diseases. However, its overuse or abuse can likely cause diarrhea, imbalance of bacterial flora, and other

symptoms, thus affecting the recovery process of the disease ${ }^{11}$. Particularly, the use of antibiotics has been associated with the dysbiosis of gut microbiota. Gut microbiota has been proven to have significant relationships with digestive tract diseases ${ }^{12}$. The development of gastrointestinal tumor, ulcerative colitis, chronic gastritis and other gastrointestinal tract associated diseases have always been along with the shifts of the diversity and composition of microbiota ${ }^{13,14}$. It has been reported that polysaccharide from the fruiting body of Hericium erinaceus could improve the relative abundance of Clostridiales,

Akkermansia and Desulfovibrio which causes a balance in the gut microbiota in colitis mice ${ }^{10}$. However, it is not clear whether the polysaccharide from the fruiting body of Hericium erinaceus could cause the recovery of diarrhea and the dysbiosis of gut microbiota induced by the abuse of antibiotic.

In this research, an antibiotic-associated diarrhea (AAD) rat model was constructed through administered lincomycin hydrochloride orally, to investigated the effects of polysaccharides from fruiting body of the fungus Hericium erinaceus on the rats with $A A D$, especially the dysbiosis of the gut microbiota, colon structure, and SCFA metabolites.

\section{Materials And Methods}

\subsection{Materials and Reagents}

Dried Hericium erinaceus fruiting body were purchased from Tonghua, Jilin, China. Lincomycin hydrochloride, CR Double-Crane Pharmaceuticals Co., Ltd. TIANamp STOOL DNA Kit (cat. No. DP328), Tiangen Biotech Co., Ltd. Acetate, propionate, and butyrate, Sigma-Aldrich. All other chemicals and reagents were purchased from Sinopharm group.

\subsection{Extraction Procedures and Physiochemical Analysis of Hericium erinaceus Polysaccharide}

Dried Hericium erinaceus fruiting body $(500 \mathrm{~g})$ were boiled in $10 \mathrm{~L}$ of distilled water for $3 \mathrm{~h}$. Then filtrated with a gauze (with 100 mesh), and the residue was used to repeat the extracting step above twice. The extracting solution was concentrated to $1.5 \mathrm{~L}$ at a temperature of $60^{\circ} \mathrm{C}$, and centrifuged (at a speed of $4500 \mathrm{rpm}$, for a period of $10 \mathrm{~min}$ ). The supernatant was combined with $6 \mathrm{~L}$ anhydrous ethanol for $12 \mathrm{~h}$ and centrifuged (at a speed of $4500 \mathrm{rpm}$, for a period of $10 \mathrm{~min}$ ). Dissolved the precipitates in $1 \mathrm{~L}$ distilled water, then treated with addition of $4 \mathrm{~L}$ anhydrous ethanol. Then dissolved precipitates in $500 \mathrm{~mL}$ distilled water, shaken thrice with $500 \mathrm{~mL}$ Sevag reagent (chloroform: $n$-butyl alcohol [V: V] = 1:5) to remove the protein. The water-layer was collected and freeze-dried, and Hericium erinaceus polysaccharides (WHP) were obtained. The monosaccharide composition, uronic acid and carbohydrate contents were analyzed as previous reports ${ }^{15,16}$. Protein content was analyzed by NDA 701 Dumas nitrogen analyzer.

\subsection{Experimental Design}


Animal experiments were carried out in accordance with the relevant guidelines formulated by Animal Care and Use Committee of Institute of Special Animal and Plant Sciences, Chinese Academy of Agricultural Sciences, while approved by the current protocol (tcs-01-335). All experiments and methods were followed the recommendations of Animal Research: Reporting of In Vivo Experiments (ARRIVE) guidelines. We declared that all methods were carried out in accordance with the relevant guidelines and regulations.

Wistar rats $(180 \pm 20 \mathrm{~g})$ were purchased from Changsheng Laboratory Animal Technology Co., Ltd, which lived at $22 \pm 0.5^{\circ} \mathrm{C}$ temperature, $50 \pm 5 \%$ humidity, and $12 \mathrm{~h}: 12 \mathrm{~h}$ light: dark cycles circumstances, all had diet and water freely. Rats were randomly divided to four groups (6/group), seperately, the control (C) group, AAD (DM) group, natural recovery (NR) group, and WHP treatment (WHP) group after acclimation. Lincomycin hydrochloride $(10 \mathrm{~mL} / \mathrm{kg})$ were gavaged twice a day to rats for 4 days to establish AAD model orally except $\mathrm{C}$ group which received same volume of physiological saline. After treated with antibiotic, rats of the DM group were anesthetized, the blood samples, caecal contents, and colon samples were obtained as before ${ }^{17}$. Then rats of WHP group treated with WHP $(100 \mathrm{mg} / \mathrm{kg})$ twice a day for 7 days, whereas other groups received same amount of physiological saline. The same methods were used to collected the samples as above.

The disease activity index (DAI) was an importand indicator to evaluate the severity of IBD ${ }^{18}$. The DAI score is the average of the following three scores: (I) weight loss (0: none; $1: 1-5 \% ; 2: 5-10 \% ; 3: 10-15 \%$; 4: >15\%), (II) stool consistency (0: normal; 1 and 2: loose; 3 and 4: diarrhea), (III) stool blood (0: normal; 1 : $+; 2: \pm ; 3:++; 4:>++)$.

\subsection{Histological Analysis}

Colon samples were treated as our previous study ${ }^{17}$ for histological observation.

\subsection{Microbiota Analysis}

Deoxyribonucleic acid (DNA) pretreated methods were performed as previously reported ${ }^{17}$.

R software (ver. 3.2.0), and the Greengenes database ${ }^{19}$ were used to compared the operational taxonomic units. The a diversity of ACE index was calculated to compare the diversity of gut microbiota ${ }^{20,21}$. The $\beta$ diversity was showed using principal component analysis (PCA) ${ }^{22}$ to identify microbiota variations. Partial Least Squares Discriminant Analysis (PLS-DA)was used to identify the inter-group differences between species ${ }^{23}$. The abundance of taxa at phylum level among samples or groups were compared ${ }^{24}$. Lefse was used to find the key species based on the linear discriminant analysis ${ }^{25}$. All raw sequences were deposited into the NCBI Sequence Read Archives.

\subsection{Measurement of SCFAs}

The SCFAs in caecal contents of each rat were analyzed as previously reported ${ }^{26}$. 


\subsection{Statistical Analysis}

Statistical analyses were performed using Prism 5 Software. All data were expressed as means \pm standard deviation (S.D.). Comparisons between groups were performed using the one-way analysis of variance or t-test. Differences were considered significant at $p<0.05$.

\section{Results}

The yield of WHP was $7.5 \%(\mathrm{w} / \mathrm{w})$. The total carbohydrate contents was $95.6 \%$, and protein content was $1.61 \%$. WHP was seen to be composed of glucose (88.6\%), galactose $(9.1 \%)$, and fructose $(2.3 \%)$. There was no uronic acid contained.

\subsection{Normal Status and DA/ scores}

The rats with $A A D$ showed loss of appetite, increased water consumption, increased frequency of defecation, and diarrhea. Compared with those in group $C$, the rats of group DM showed decrease in body weight, but increased water intake, suggesting the AAD rat model was successful established. The DAI reflected the diarrhea status of the AAD rats. A lower DAl value indicates a better recovery status. As shown in Table 1, WHP decreased the DAI significantly compared with the NR and DM groups, suggested that WHP obviously alleviated the diarrhea status and gut injury resulting from lincomycin hydrochloride.

\subsection{Effects of WHP on Colon Structure}

HE staining was the effective way to identify the morphology changes of the colon (Fig. 1). Colon of group $\mathrm{C}$ had normal histological structural features, which had a uniform and smooth intestinal villi. After treated with antibiotics, morphological damage was shown in the colons of group DM, mainly in the form of shorter intestinal villi, rough mucosal surface, and inflammatory infiltration. The group WHP showed improving effects in morphological structure compared to group NR, whereby the longer and denser intestinal villi, the smoother mucosal surface, more compact intestinal epithelial cells, and the reduced intestinal mucosal edema and cell shedding, the increased globet cells.

\subsection{Effects of WHP on Gut Microbiota}

\subsubsection{Diversity of Gut Microbiota}

We used ACE index analysis to identify the differences in the richness and diversity of gut microbiota. It was suggested that the richness significantly decreased in group DM compared with group $C$. As expected, the ACE index was recovered in group NR and group WHP (Figure. 2A). There were significant differences between rats of groups $C$ and $D M$, as well as groups $C$ and NR. Interestingly, it was not shown significant difference between groups $\mathrm{C}$ and WHP, suggesting that WHP could improve the recovery of the richness of the gut microbiota nearer to group $C$ than physiological saline. It was also shown in the Venn analysis (Figure. 2B) that the total and unique OUT in groups C, DM, NR, WHP were accordance with the results of $A C E$ index. 
PCA and PLS-DA analysis were used to analyze similarities that existed in microbiota between groups. DM group showed great shift on microbiota communities compared with the group $\mathrm{C}$, however, after treatment with physiological saline or WHP, there were some recovery tendency (Fig. 2C, 2D). According to PCA, the structure of micro flora community reduced which was had similarity between groups $C$ and DM, suggesting the structural changes after antibiotic treatment. However, the structure of micro flora community of group NR and group WHP were similar and close to the group C. PLS-DA results was consistent with PCA, which showed that the microbial communities structure of each group was relative independent, and group NR and group WHP were recovered following antibiotics destruction.

\subsubsection{Composition Shifts of Gut Microbiota}

C, DM, NR, and WHP groups showed significant changes in the composition and amounts of gut microbial species. The four groups were mainly composed of Firmicutes, Bacteroidetes, and Proteobacteria (Fig. 3A) at the phylum level. The relative abundance of Bacteroidetes was significant decrease in rats of DM group, while Firmicutes and Proteobacteria showed dramatic increase. The results in the composition of the gut microbiota as seen between the DM and $\mathrm{C}$ group suggested the successful construction of the AAD model which along with the microbiota dysbiosis. The Bacteroidetes and Firmicutes recovered to their normal levels, while Proteobacteria still increased in group NR and WHP.

The gut microbiota composition showed great shift among the four groups at the genus level (Fig. 3B). The relative abundances of Coprococcus and Pesudomonas were significantly increased, while Lactobacillus and Oscillospira were decreased in group DM compared with group C, respectively. In the groups NR and WHP, the relative abundance of Coprococcus, Lactobacillus, and Oscillospira were seen to have been recovered to the level of Group C. Compared with group NR, the levels of Lactobacillus and Butyricicoccus significantly increased, while Enterococcus and Allobaculum were decreased in group WHP (Fig. 3B). Although the abundance of Lactobacillus was increased both in NR and WHP groups compared with DM group, WHP treatment was more efficient than that of physiological saline.

\subsection{SCFAs Production}

Acetate, propionate, and butyrate were measured to investigated the effects of WHP on microbial metabolites (Fig. 4). Caecal contents of group DM revealed decreased concentrations of acetate, propionate, and butyrate compared with that of group C. Group NR and group WHP could be recovered from the acetate and propionate production significantly. The level of butyrate significantly increased in the group WHP compared with group NR $(p<0.05)$, while acetate and propionate didn't show obvious changes.

\subsection{Effects of WHP on the Functional analysis of the gut microbiota}

The data (Fig. 5) showed that the gut microbiota mainly affected that energy, carbohydrate and amino acid metabolic processes. DM group showed a decreased energy and carbohydrate metabolic rate, and an increased amino acid metabolic rate. Compared with NR group, the WHP group of the amino acid 
metabolic processes were increased, but the energy and carbohydrate metabolic processes were decreased.

\section{Discussion}

Diarrhea and colon structure destruction have been the common side effects of excessive antibiotics intake. Our results found that along with the misuse of antibiotics, the intestinal structure of rats had certain ability to undergo repair, however, the intervention of WHP could enhance the effects above, decreased the inflammatory infiltration, and protect the colon structure faster and more effectively, as well as the sufficient improvement of the diarrhea status.

According to the result of showing the analysis in diversity of the gut microbiota, both NR and WHP could recover from the damage caused by antibiotics to a certain extent. But WHP was more orderly than NR in the recovery period; the abundance and diversity of gut microbiota were closer to that of normal mice, especially the diversity index based on ACE. The composition of gut microbiota in group NR had little change compared with WHP at the phylum level, mainly because of the phylum level ranged widely in taxonomy, and each that phylum contains many members, it was not easy to show changes. However, at the genus level, the disruption caused by antibiotics not only reduces the number of bacteria, but also changes their composition. The most obvious was that WHP could promote the relative abundance of Lactobacillus and Butyricicoccus, whereas decreased of Allobaculum and Enterococcus. Lactobacillus was recognized as beneficial bacterium, which has a positive role in promoting human health, such as conqure with the infections diarrhea 27,28 . The Butyricicoccus bacteria are a genus contained in many probiotics, which has always displayed lower level in fecal samples of IBD patients ${ }^{29}$. Butyricicoccus was related to the production of butyrate, a type of SCFAs showed beneficial effects to the host. Allobaculum and Enterococcus were related with the gut microbiota dysbiosis. Although Enterococcus is no longer considered to be a typical food borne pathogen, but its presence often raises health concerns. At the same time, some strains in the Enterococcus genus were proved to possesses resistance to many antibiotics, and play an important role in infections, such as nosocomial infections, bacteremia, endocarditis ${ }^{30}$. So the decreasing effects of WHP on Enterococcus were seemed to be beneficial to the host. So the improving effects of WHP on the AAD might be correlated with the ability of adjust the relative abundance of Lactobacillus, Butyricicoccus, Allobaculum, and Enterococcus, which are all associated with the health of the gastrointestinal tract.

To research the different influences of the various polysaccharides on AAD, we used the same method and compared the result with the polysaccharides derived from Schisandra chinensis (WSP) ${ }^{26}$ and Astragalus membranaceus (WAP) ${ }^{31}$. When compared with physiological saline treatment, WSP could promote the relative abundance of Intestinibacter, Blautia, and LachnospiraceaeUCG-008, but reduced that of RuminococcaceaeUCG-014, Ruminococcus-1, and Erysipelatoclostridium. WAP could increase the relative abundance of Pseudomonas, but decreased Allobaculum and Coprococcus abundance at the genus level. At the same time, there are some similarities in the effects on some species. For example, 
both WHP and WAP can reduce the abundance of Allobaculum. These results suggest that different sources of polysaccharides showed various effects on gut microbiota, which may be related to the differences of monosaccharide composition, molecular weight, length of the main chain, space conformation and so on.

It has been reported ${ }^{10}$ that polysaccharide from the fungus Hericium erinaceus could improve the relative abundance of Clostridiales, Akkermansia and Desulfovibrio which would in turn cause a balance in the gut microbiota in mice with colitis. However, in our study, WHP was not found to have an effect on the abundance of these floras. This may be because the two animal models are different, which possesses different influences on the gut microbiota, and also indirectly affect the effect of Hericium erinaceus polysaccharides on the dysbiosis of gut microbiota. Therefore, animal models are also an important consideration of the research on the applications of plant polysaccharides on gut microbiota.

Butyrate is one of the energy sources of the colonocytes. It could modulates the gut barrier protection through inducing the consumption of $\mathrm{O}_{2}$ in the epithelium ${ }^{32}$. In terms of metabolites, the significant ability of butyrate production of WHP attracted our attention because of the multiple beneficial effects of butyrate, such as decrease the pro-inflammatory cytokines; increase the learning and memory abilities in neurodegenerative diseases, and the abolishment of lipopolysaccharide induced depressive-like behaviors by the decrease in microglia activation ${ }^{33}$. The increase in butyrate production might be due to the fact that WHP contains a large amount of glucose, which could be composed of glucan and in turn serve as the function of a probiotic and could be fermented by the gut microbiota, supply for metabolism, and utilization, then further promote the abundance of butyric acid-producing bacteria, such as the Butyricoccus.

The Chinese herbal medicine had a long history of application in China, which had the characteristics of natural and non-toxic ${ }^{34}$, but the mechanisms of some activities were not clear. The research on relationships between gut microbiota and herbal medicine provided a novel focus point for the development of Chinese herbal medicines. In particular, some medicinal food was the first choice for daily health care. In China, the fruiting body of Hericium erinaceus often appeared in the daily diet used as a delicacy or medicine for protecting the gastrointestinal tract thereby promoting the digestion of food. The protective effect on gastrointestinal tract was often closely connected to gut microbiota. From the results of this study, Hericium erinaceus polysaccharide could promote the imbalance caused by the destruction of the gut microbiota caused due to antibiotics, improve the level of metabolites, and thus alleviate AAD. However, there is still need for more experiments to confirm the potential molecular mechanism of the effects of WHP on AAD.

\section{Conclusion}

In conclusion, WHP had beneficial effects on the rats with AAD induced by lincomycin hydrochloride through recovering the colon damage, improving the gut microbiota dysbiosis, and promoting the SCFAs level. WHP could adjust the relative abundance of Lactobacillus, Butyricicoccus, Allobaculum and 
Enterococcus at the genus level and promoted the producing of metabolites butyrate. WHP might use as a functional component to relieved the AAD depending on its ability to modulate the gut microbiota and homeostasis.

\section{Declarations}

\section{Acknowlegments}

This research was funded by Scientific and Technologic Foundation of Jilin Province, grant number 20200201116JC and Young PhD funding of Jilin Agricultural Science and Technology University, grant number 2021-7011.

\section{Conflicts of interest}

The authors declare that there is no conflict of interest.

\section{Author contributions}

data curation, D.D. Ren; resources, S.C. Tan; methodology, Y.L. Qi; Conceptualization, writing - original draft and writing - review \& editing, S.S. Li; supervision and project administration, Y.S. Sun. All authors reviewed the manuscript.

\section{Data availability statement}

The datasets used during the current study available from the corresponding author on reasonable request.

\section{References}

1. He, X. R., et al. Structures, biological activities, and industrial applications of the polysaccharides from Hericium erinaceus (Lion's Mane) mushroom: A review. Int. J. Biol. Macromol. 97, 228-237 (2017). doi: 10.1016/j.ijbiomac.2017.01.040

2. Zhu, Y., et al. Optimization of enzyme-assisted extraction and characterization of polysaccharides from Hericium erinaceus. Carbohyd. Polym. 101, 606-613 (2014). doi:

10.1016/j.carbpol.2013.09.099

3. Yan, J. K., et al. Comparative study of physicochemical properties and bioactivity of Hericium erinaceus polysaccharides at different solvent extractions. Carbohyd. Polym. 193, 373-382 (2018). doi: 10.1016/j.carbpol.2018.04.019

4. Wang, M., Gao, Y., Xu, D., \& Gao, Q. A polysaccharide from cultured mycelium of Hericium erinaceus and its anti-chronic atrophic gastritis activity. Int. J. Biol. Macromol. 81, 656-661 (2015). doi: 10.1016/j.ijbiomac.2015.08.043 
5. Liao, B. W., \& Huang, H. H. Structural characterization of a novel polysaccharide from Hericium erinaceus and its protective effects against $\mathrm{H}_{2} \mathrm{O}_{2}$-induced injury in human gastric epithelium cells. J. Funct. Foods 56, 265-275 (2019). doi: 10.1016/j.jff.2019.03.028

6. Wang, X. Y., et al. Gastroprotective activity of polysaccharide from Hericium erinaceus against ethanol-induced gastric mucosal lesion and pylorus ligation-induced gastric ulcer, and its antioxidant activities. Carbohyd. Polym. 186, 100-109 (2018). doi: 10.1016/j.carbpol.2018.01.004

7. Feng, T., et al. Hericium Erinaceus $\beta$-glucan modulates in vitro wheat starch digestibility. Food Hydrocolloid. 96, 424-432 (2019). doi: 10.1016/j.foodhyd.2019.05.044

8. Sheu, S. C., Lyu, Y., Lee, M. S., \& Cheng, J. H. Immunomodulatory effects of polysaccharides isolated from Hericium erinaceus on dendritic cells. Process Biochem. 48, 1402-1408 (2013). doi: 10.1016/j.procbio.2013.06.012

9. Wang, X. Y., Yin, J. Y., Nie, S. P., \& Xie, M. Y. Isolation, purification and physicochemical properties of polysaccharide from fruiting body of Hericium erinaceus and its effect on colonic health of mice. Int. J. Biol. Macromol. 107, 1310-1319 (2018). doi: 10.1016/j.ijbiomac.2017.09.112

10. Ren, Y. L., et al. Polysaccharide of Hericium erinaceus attenuates colitis in C57BL/6 mice via regulation of oxidative stress, inflammation-related signaling pathways and modulating the composition of the gut microbiota. J. Nutr. Biochem. 57, 67-76 (2018). doi:

10.1016/j.jnutbio.2018.03.005

11. Allen, S. J., et al. A high-dose preparation of lactobacilli and bifidobacteria in the prevention of antibiotic-associated and Clostridium difficile diarrhoea in older people admitted to hospital: A multicentre, randomised, double-blind, placebo-controlled, parallel arm trial. Health Technol. Asses. 17, 1-140 (2013). doi: 10.3310/hta17570

12. Jones, R. M. The influence of the gut microbiota on host physiology: In pursuit of mechanisms. Yale J. Biol. Med. 89, 285-297 (2016).

13. Thomas, S., et al. The host microbiome regulates and maintains human health: A primer and perspective for Non-Microbiologists. Cancer Res. 77, 1783-1812 (2017). doi: 10.1158/00085472.CAN-16-2929

14. Marchesi, J. R., et al. The gut microbiota and host health: A new clinical frontier. Gut, 65, 330-339 (2016). doi: 10.1136/gutjnl-2015-309990

15. Hua, M., et al. Structure, physicochemical properties and adsorption function of insoluble dietary fiber from ginseng residue: A potential functional ingredient. Food Chem. 286, 522-529 (2019). doi: 10.1016/j.foodchem.2019.01.114

16. Huang, X. J., et al. An antioxidant a-glucan from Cladina rangiferina (L.) Nyl. and its protective effect on alveolar epithelial cells from $\mathrm{Pb}^{2+}$-induced oxidative damage. Int. J. Biol. Macromol. 112, 101109 (2018). doi: 10.1016/j.ijbiomac.2018.01.154

17. Wang, L. F., et al. Injected laquinimod D-a-tocopheryl polyethylene glycol-1000 succinate polymeric micelles for the treatment of inflammatory bowel disease. Colloid. Surface. B 185, 110575 (2020). doi: 10.1016/j.colsurfb.2019.110575 
18. Li, S. S., et al. Effects of Panax ginseng polysaccharides on the gut microbiota in mice with antibiotic-associated diarrhea. Int. J. Biol. Macromol. 124, 931-937 (2019). doi:

10.1016/j.ijbiomac.2018.11.271

19. Blaxter, M., et al. Defining operational taxonomic units using DNA barcode data. Philos. Trans. R. Biol. Sci. 360, 1935-1943 (2005). doi: 10.1098/rstb.2005.1725

20. Heck, K. L., Belle, G. V., \& Simberloff, D. Explicit calculation of the rarefaction diversity measurement and the determination of sufficient sample size. Ecology, 56, 1459-1461 (1975). doi:

$10.2307 / 1934716$

21. Kemp, P. F., \& Aller, J. Y. Bacterial diversity in aquatic and other environments: What $16 S$ rDNA libraries can tell us. FEMS Microbiol. Ecol. 47, 161-177 (2004). doi: 10.1016/S0168-6496(03)002575

22. Ramette, A. Multivariate analyses in microbial ecology. FEMS Microbiol. Ecol. 62, 142-160 (2007). doi: 10.1111/j.1574-6941.2007.00375.x

23. Chen, Y. F., et al. Characterization of fecal microbial communities in patients with liver cirrhosis, Hepatology, 54, 562-572 (2011). doi: 10.1002/hep.24423

24. White, J. R., Nagarajan, N., \& Pop, M. Statistical methods for detecting differentially abundant features in clinical metagenomic samples. Plos Comput. Biol. 5, e1000352 (2009). doi: 10.1371/journal.pcbi.1000352

25. Segata, N., et al. Metagenomic biomarker discovery and explanation. Genome Biol. 12, R60 (2011). doi: 10.1186/gb-2011-12-6-r60

26. Qi, Y. L., et al. Effects of Schisandra chinensis polysaccharides on rats with antibiotic-associated diarrhea. Int. J. Biol. Macromol. 124, 627-634 (2019). doi: 10.1016/j.jjbiomac.2018.11.250

27. Gibson, G. R., Probert, H. M., Loo, J. V., Rastall, R. A., \& Roberfroid, M. B. Dietary modulation of the human colonic microbiota: updating the concept of prebiotics. Nutr. Res. Rev. 17, 259-275 (2004). doi: 10.1079/NRR200479

28. Wang, Y., et al. Antioxidant properties of probiotic bacteria. Nutrients 9, 521 (2017). doi: $10.3390 /$ nu9050521

29. Geirnaert, A., et al. Butyricicoccus pullicaecorum, a butyrate producer with probiotic potential, is intrinsically tolerant to stomach and small intestine conditions. Anaerobe 30, 70-74 (2014). doi: 10.1016/j.anaerobe.2014.08.010

30. Kim, N. H., et al. Virulence patterns and prevalence of seven Enterococcus species isolated from meats and leafy vegetables in South Korea. Food Control 108, 106867 (2020). doi: 10.1016/j.foodcont.2019.106867

31. Li, S. S., Qi, Y. L., Ren, D. D., Qu, D., \& Sun, Y. S. The structure features and improving effects of polysaccharide from Astragalus membranaceus on antibiotic-associated diarrhea. Antibiotics-Basel 9, 8 (2020). doi: 10.3390/antibiotics9010008

32. Kelly, C. J., et al. Crosstalk between microbiota-derived short-chain fatty acids and intestinal epithelial HIF augments tissue barrier function. Cell Host Microbe 17, 662-671 (2015). doi: 
10.1016/j.chom.2015.03.005

33. Matt, S. M., et al. Butyrate and dietary soluble fiber improve neuroinflammation associated with aging in mice. Front. Immunol. 9, 1832 (2018). doi: 10.3389/fimmu.2018.01832

34. Ren, S., et al. Ginsenoside Rb1, A major saponin from Panax ginseng, exerts protective effects against aetaminophen-induced hepatotoxicity in mice. Am. J. Chin. Med. 67, 10342-10351 (2019). doi: 10.1142/S0192415X19500927

\section{Tables}

Table 1

Disease activity index (DAl) scores

\begin{tabular}{|ll|}
\hline Group & DAI \\
\hline Con & $0.0 \pm 0.0$ \\
\hline DM & $3.1 \pm 0.4^{\mathrm{a}}$ \\
\hline NR & $2.5 \pm 0.4^{\mathrm{a}}$ \\
\hline WHP & $0.7 \pm 0.1^{\mathrm{b}}$ \\
\hline a-b Data within a column with different superscripts differed significantly $(p<0.05)$. \\
\hline
\end{tabular}

\section{Figures}



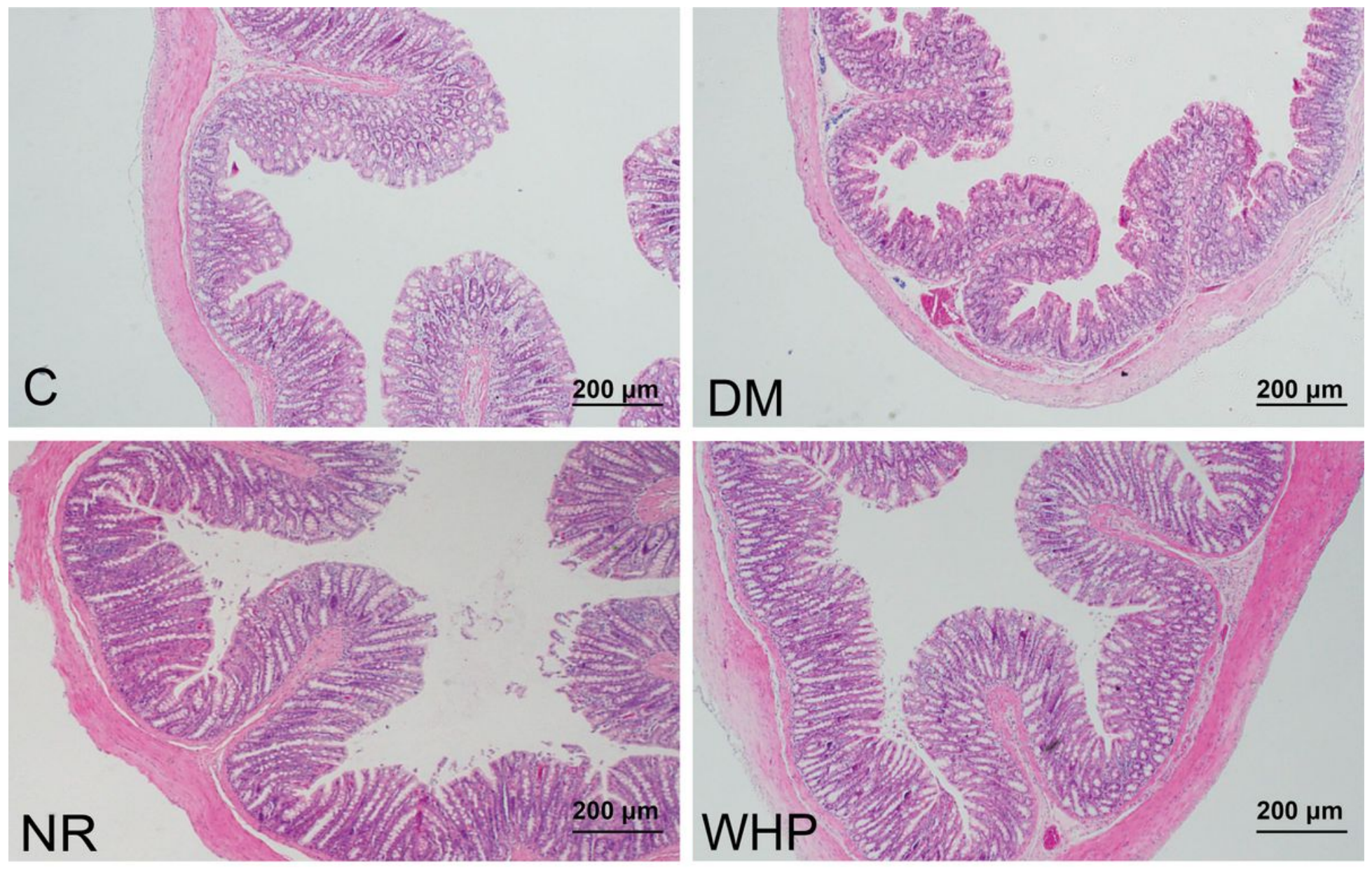

\section{Figure 1}

Histological analysis of colon structure (40x). C, control group; DM, antibiotic-associated diarrhea group; NR, natural recovery group; WHP, Hericium erinaceus polysaccharide group. 
A

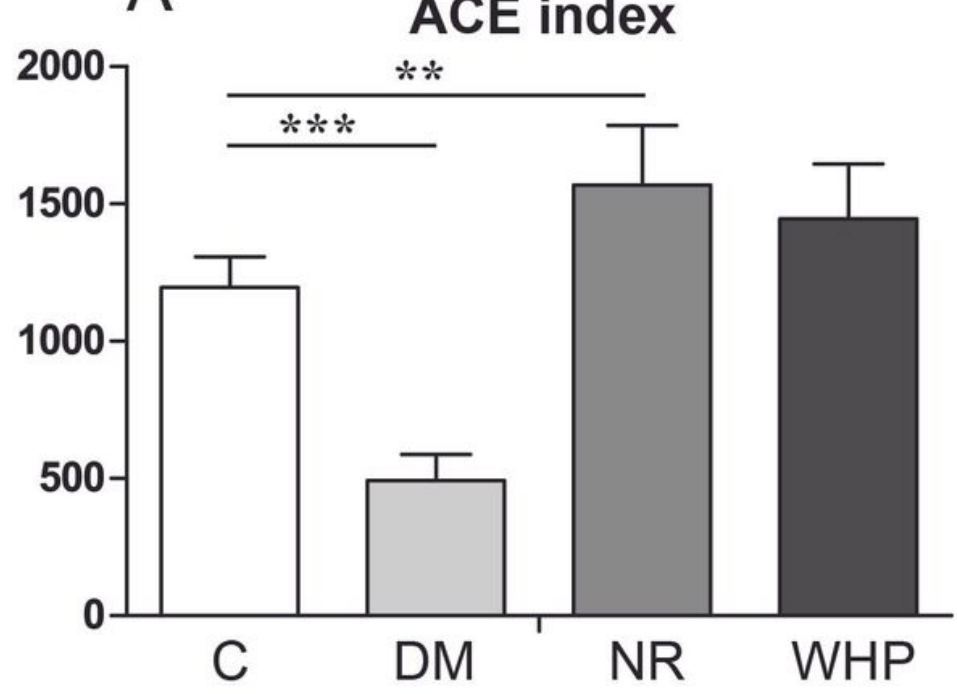

C

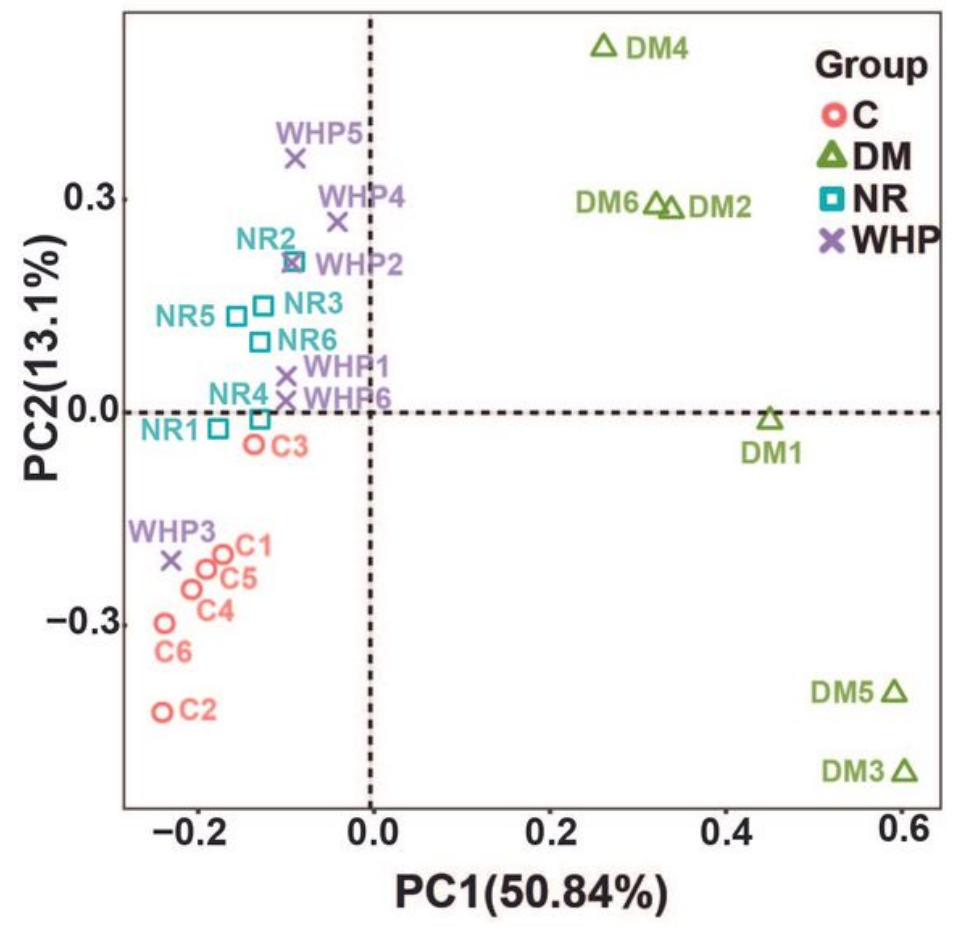

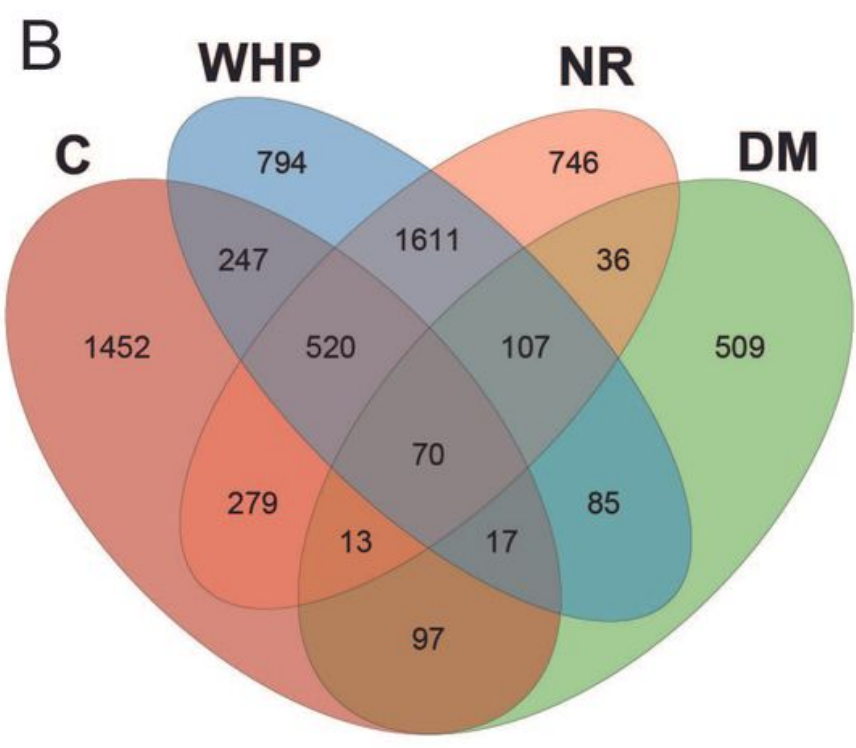
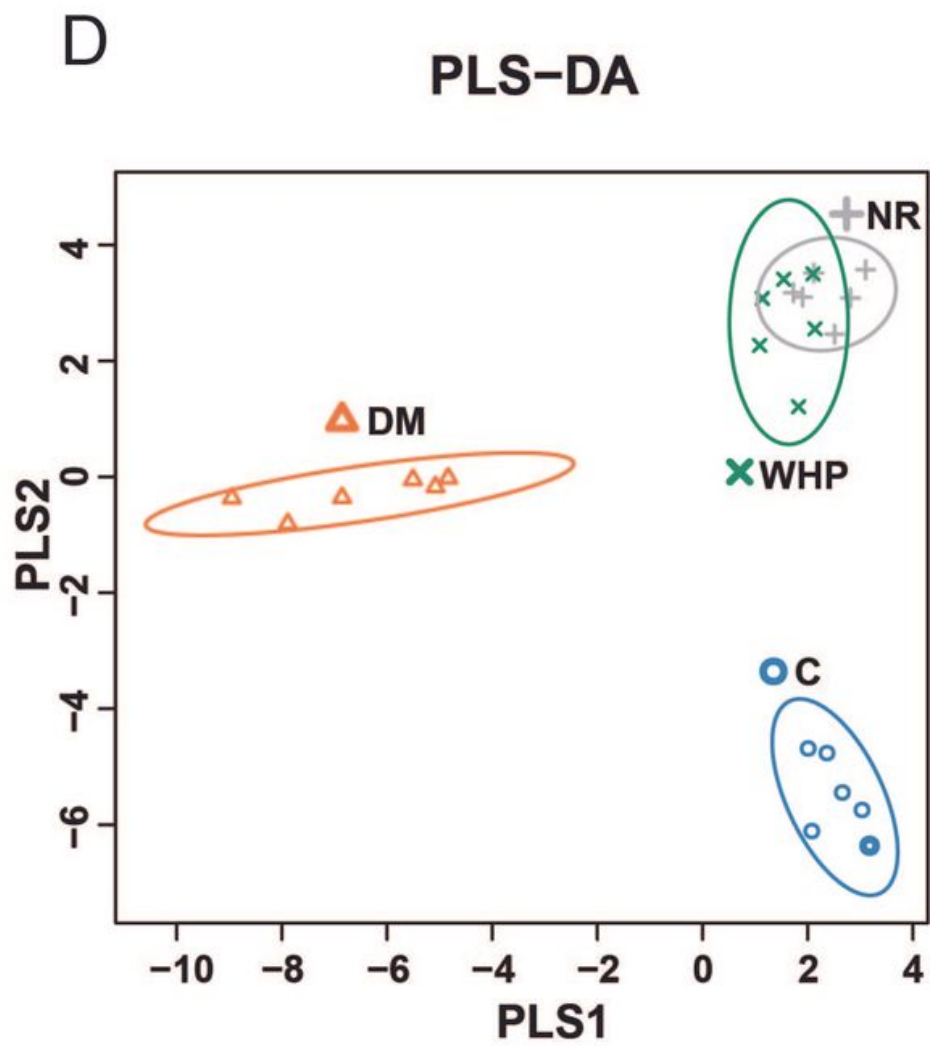

Figure 2

Diversity analysis of the gut microbiota. (A) ACE index, (B) Venn analysis, (C) PCA analysis, (D) PLS-DA analysis. C, control group; DM, antibiotic-associated diarrhea group; NR, natural recovery group; WHP, Hericium erinaceus polysaccharide group. Data are expressed as means \pm S.D. $(n=6)$. ${ }^{\star *}, p<0.01 ; * \star \star, p$ $<0.001$. 


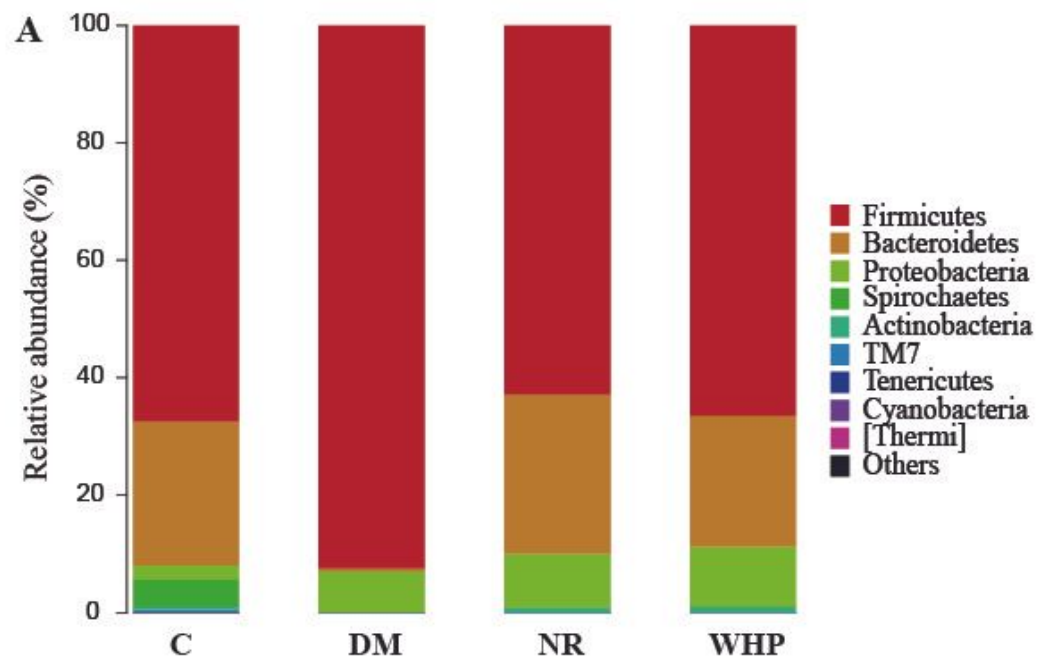

B

Lactobacillus

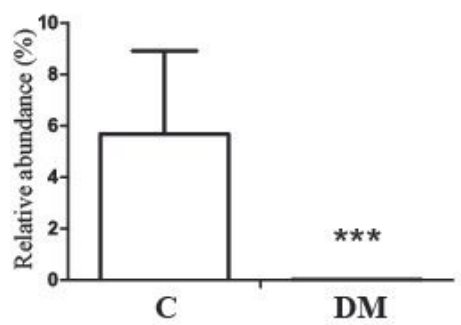

Lactobacillus

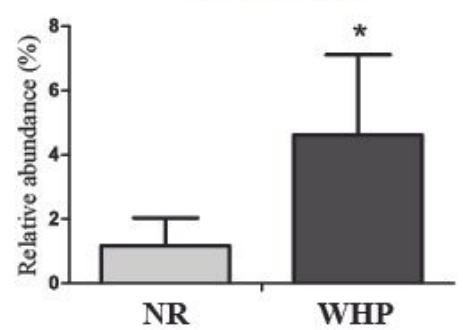

Oscillospira

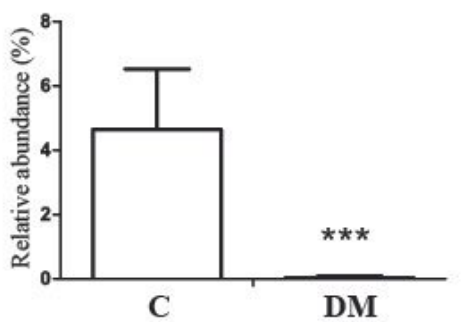

Butyricicoccus

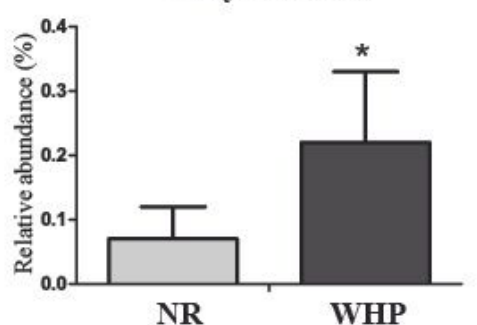

Coprococcus

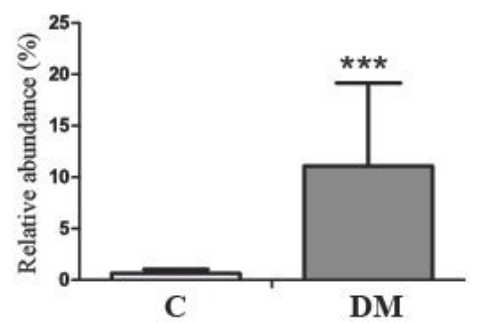

Enterococcus

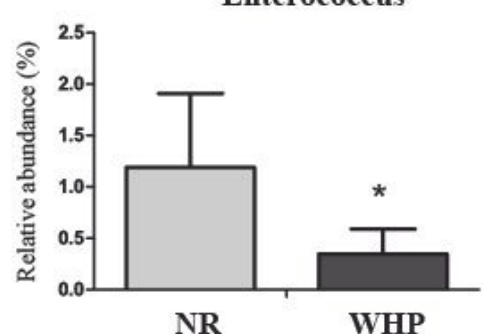

Pseudomonas

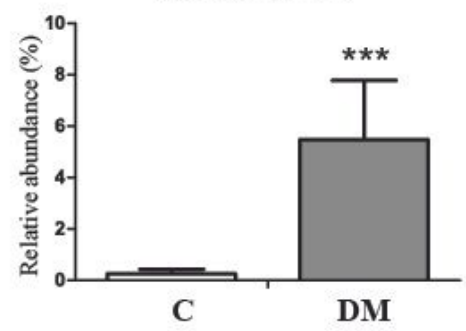

Allobaculum

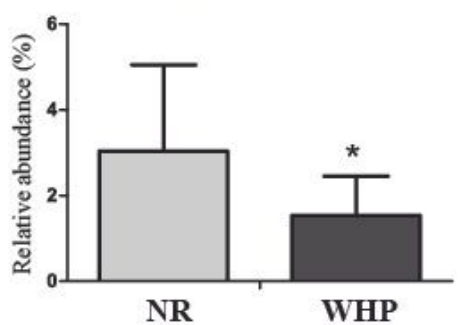

Figure 3

The composition changes of gut microbiota. (A) Phyla level; (B) Genus level. C, control group; DM, antibiotic-associated diarrhea group; NR, natural recovery group; WHP, Hericium erinaceus polysaccharide group. ${ }^{*} p<0.05 ; * \star * p<0.001$ 
Acetate

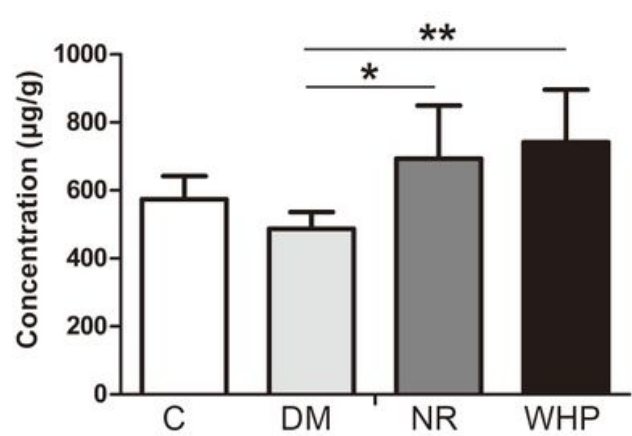

Propionate

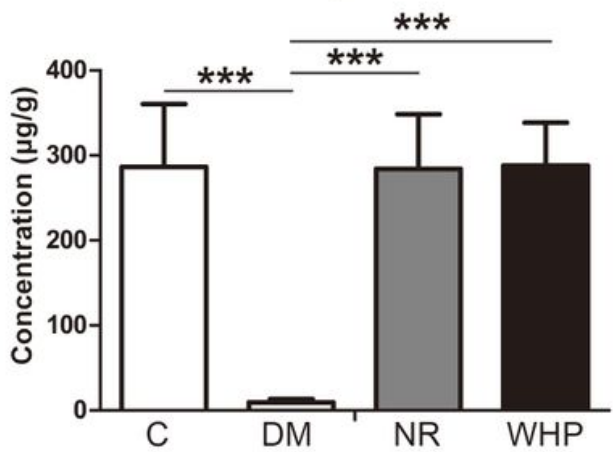

Butyrate

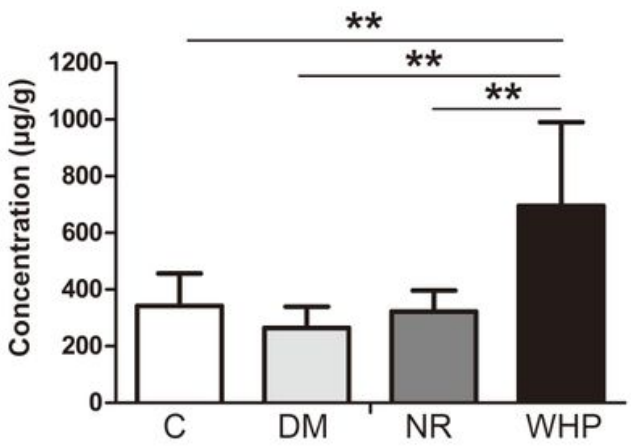

Figure 4

SFCA concentration analysis in caecal contents. C, control group; DM, antibiotic-associated diarrhea group; NR, natural recovery group; WHP, Hericium erinaceus polysaccharide group. Data are expressed as means \pm S.D. $(n=6) .{ }^{*} p<0.05 ; * \star p<0.01$.

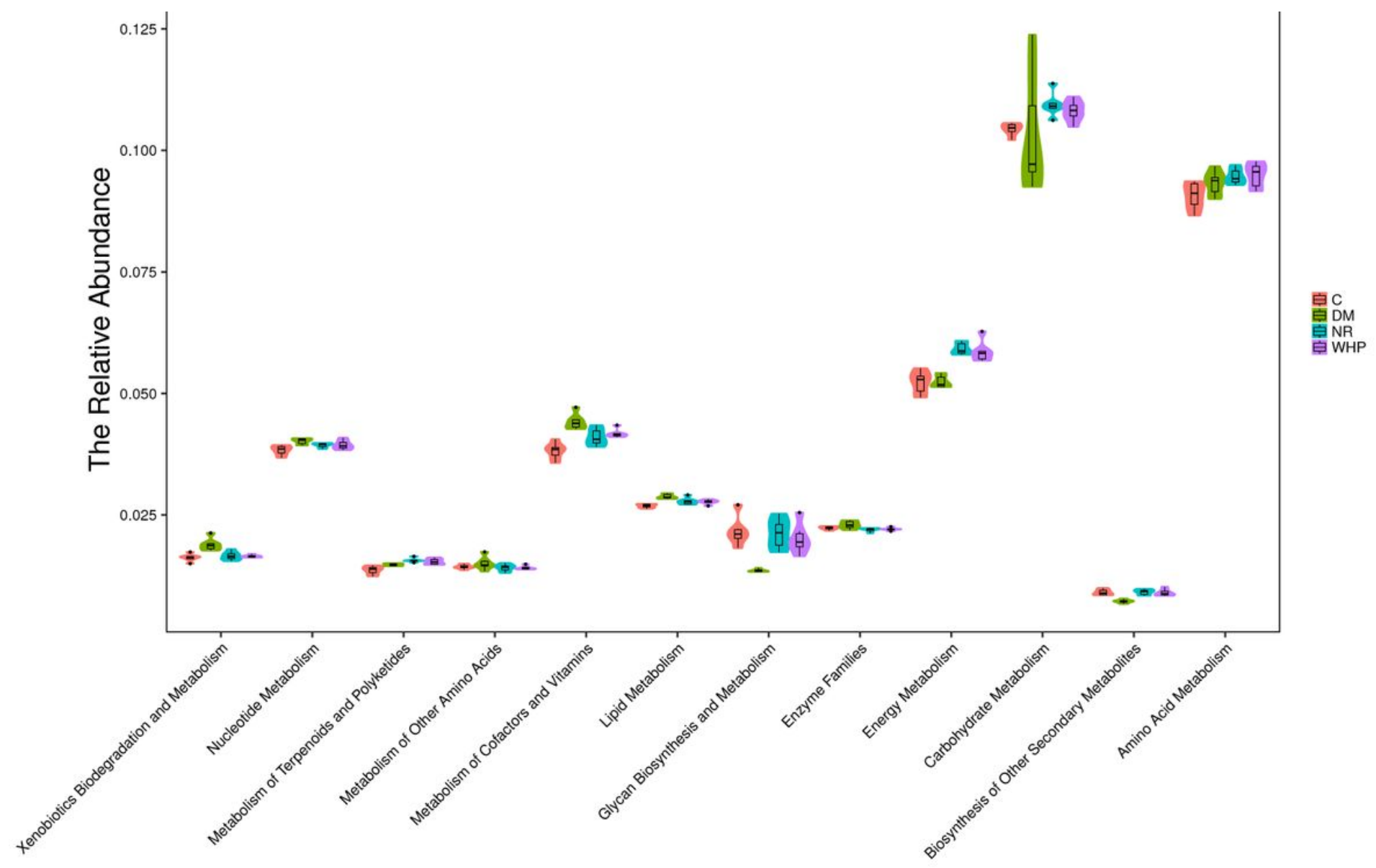

Figure 5

Effects of WHP on metabolic processes. Where: $\mathrm{C}$, is the control group; DM, is the antibiotic-associated diarrhea group; NR, the natural recovery group; WHP, The Hericium erinaceus polysaccharide group. 